DOI: $10.35643 /$ Info.24.2.2

Artículo Original

\title{
La teoría epidémica aplicada a la idea de sustentabilidad en las áreas de Ecología y Ordenamiento territorial ${ }^{1}$
}

\section{The epidemic theory applied to the concept of sustainability in the areas of Ecology and Territorial Planning}

\section{Patricia Machín Faral ${ }^{1}$}

${ }^{1}$ Facultad de Información y Comunicación. Universidad de la República. Correo electrónico pmachin01@gmail.com. ORCID: 0000-0003-2910-0649

\section{Resumen}

Se aplica la teoría epidémica de William Goffman y Vaun A. Newill, inscrita dentro de los estudios bibliométricos en el área de la Ciencia de la Información, para explicar el patrón de propagación del concepto sustentabilidad en el campo científico a nivel internacional. Para ello, se toman los autores de artículos de publicaciones científicas de Ecología y Ordenamiento territorial indizadas en la plataforma de información Web of Science, desde el año 1987 hasta 2015 que contienen el concepto de sustentabilidad como tema.

Entre las principales conclusiones a las que se arriba se destaca la existencia de una epidemia del concepto de sustentabilidad en el área de la Ecología y el Ordenamiento territorial, la tendencia creciente del tema a nivel internacional y la pertinencia del modelo de Goffman como un indicador de suma importancia para determinar la existencia de una epidemia.

Palabras clave: ANÁLISIS BIBLIOMÉTRICO, TEORÍA EPIDÉMICA, SUSTENTABILIDAD

\begin{abstract}
The epidemic theory of William Goffman and Vaun A. Newill, inscribed within the bibliometric studies in the area of Information Science, is applied to explain the propagation pattern of the concept of sustainability in the scientific field at international level. For this, the authors of articles of scientific publications of Ecology and Territorial Planning indexed in the Web of Science information platform are taken, from 1987 to 2015 that contain the concept of sustainability as a theme.
\end{abstract}

\footnotetext{
${ }^{1}$ Síntesis de la Tesis de Maestría en Información y Comunicación defendida en agosto de 2018 para obtener el título de Magíster en la Facultad de Información y Comunicación de la Universidad de la República, Montevideo, Uruguay.
} 
Among the main conclusions mentioned above is the existence of an epidemic of the concept of sustainability in the area of Ecology and Land Management, the growing trend of the issue at the international level and the relevance of the Goffman model as an indicator of very important to determine the existence of an epidemic.

\section{Key words: BIBLIOMETRIC ANALYSIS, EPIDEMIC THEORY, SUSTAINABILITY}

Fecha de recibido: 17/07/2019

Fecha de aceptado: 05/10/2019

\section{Introducción}

El tema de sustentabilidad se ha difundido rápidamente en los últimos años en la literatura científica y en publicaciones de todo tipo, incluso fue elegida como una de las palabras de la década 2000-2009 por la organización Global Language Monitor.

Se propone la teoría epidémica de William Goffman y Vaun A. Newill para explicar el patrón de propagación de ese concepto en el campo científico. Para ello, se toman los autores de artículos de publicaciones científicas de Ecología y Ordenamiento territorial indizadas en la plataforma de información Web of Science, desde el año 1987 hasta 2015 que contienen el concepto de sustentabilidad como tema. La teoría epidémica se inscribe dentro de los estudios bibliométricos, en el área de la Ciencia de la Información y utiliza modelos matemáticos para esquematizar la difusión de determinados temas o líneas de investigación.

\section{Objetivo general}

El objetivo general es identificar un patrón epidemiológico en la difusión del término "sustentabilidad" en los campos de estudio de la Ecología y el Ordenamiento Territorial en la bibliografía científica.

\section{Objetivos específicos}

- Obtener un registro de la utilización del término "sustentabilidad" como tema, aparecido en publicaciones científicas de Ecología y de Ordenamiento territorial en la plataforma de información Web of Science.

- Especificar el ritmo de utilización del término "sustentabilidad", tomando las variables de frecuencia, autores y cobertura geográfica.

- Interpretar el conjunto de datos de acuerdo a la teoría epidémica, utilizando una ecuación de análisis determinístico.

\section{MARCO TEÓRICO Y ANTECEDENTES}

- Teoría epidémica 
El primer intento de aplicar los modelos matemáticos utilizados en el área de la epidemiología al campo de las ideas fue el de los autores Goffman y Newill $(1964,225)$ en el artículo titulado "Generalization of epidemictheory: an application to the transmission of ideas". En este artículo plantean que es posible estudiar el contagio desde el punto de vista cuantitativo, como un indicador de que se está produciendo una influencia. El análisis de la transmisión de ideas como proceso epidémico fue pensado para el diseño y uso de sistemas de recuperación de información en la medida que se puede predecir dónde y cuándo una idea toma proporciones epidémicas, la duración y la intensidad de la epidemia o los artículos clásicos de una disciplina que configuran el material infeccioso inicial. El dinamismo de los sistemas de recuperación de información, reflejo de la interacción de los investigadores y la literatura, se puede beneficiar del método del enfoque epidémico para describir y predecir aspectos fundamentales de esa interacción.

La descripción y la predicción del proceso de transmisión de ideas por medio de un modelo matemático no reemplazan a un análisis completo del proceso. Goffman y Newill aclaran en la presentación de su estudio que una comprensión global requiere estudios complementarios a los matemáticos. Así como, para el caso de una enfermedad infecciosa se requieren investigaciones biológicas, ecológicas y clínicas, en la transmisión de ideas, el análisis se enriquece de forma importante con estudios psicológicos, sociológicos y lingüísticos. El modelo epidémico no puede en sí mismo explicar por qué un artículo determinado puede infectar a los lectores con una idea, puesto que no maneja todas las variables que inciden en el proceso, pero sí puede indicar la existencia de la influencia.

Una población de autores puede adoptar uno de los siguientes estados:

1- Infectados, son aquellos autores que poseen material infeccioso, es decir, que publican trabajos sobre un tema,

2- Susceptibles, son los autores que pueden infectarse por el contacto con un material infeccioso,

3- Removidos, son los autores que no forman parte de la población a estudiar porque ya no tienen interés en una idea o murieron.

En el cuadro 1 se ejemplifica la comparación entre los diferentes estados presentes en una enfermedad infecciosa, así como los que puede adoptar una persona en una epidemia intelectual.

Cuadro 1. Comparación entre las enfermedades infecciosas y una epidemia intelectual

\begin{tabular}{|c|c|c|c|c|}
\hline & Agente & Infectado & Susceptible & Removido \\
\hline $\begin{array}{l}\text { Enfermedad } \\
\text { infecciosa }\end{array}$ & Materialinfeccioso & Persona enferma & $\begin{array}{l}\text { Persona que será } \\
\text { infectada por medio } \\
\text { de un contacto }\end{array}$ & $\begin{array}{l}\text { Persona que adquiere } \\
\text { inmunidad o que } \\
\text { muere }\end{array}$ \\
\hline $\begin{array}{l}\text { Epidemia } \\
\text { intelectual }\end{array}$ & Idea & $\begin{array}{l}\text { Autor de un } \\
\text { artículo }\end{array}$ & $\begin{array}{l}\text { Lector de } \\
\text { artículo }\end{array}$ & $\begin{array}{l}\text { Persona que pierde el } \\
\text { interés en un tema o } \\
\text { que muere }\end{array}$ \\
\hline
\end{tabular}


Como ejemplos de la aplicación de esta teoría por otros autores se puede mencionar:

- Paulo da Terra Caldeira $(1975,5)$ aplicando la teoría a la literatura brasileña sobre la enfermedad de Chagas, una enfermedad con alta prevalencia y mortalidad en Brasil, con el objetivo de explicar la evolución de las investigaciones en la erradicación de ese mal así como las instituciones nacionales e internacionales que trabajan para ello,

- Bennion y Neuton $(1976,53)$ estudiando el "agua anómala" o "polywater", descubierta en 1961 por el científico ruso Fedyakin y cuyas propiedades (menor temperatura de congelación y mayor temperatura para su ebullición) la hacían diferente al agua común y originó un importante número de investigaciones al respecto (Diamond, 1988),

- $\quad$ Oliveira $(1984,25)$ analizando el comportamiento de la literatura brasileña de teología adventista, a partir de la preocupación en el medio teológico adventista por la inclusión de material extranjero en las publicaciones brasileñas especializadas en esa temática y verificar si había una participación efectiva en su desarrollo,

- $\quad$ Khelil $(2002,1)$ quien estudia la difusión de la información en las redes de dispositivos móviles o malla de nodos móviles (mobile ad hoc network o MANET) con el fin de determinar la mejor estrategia en la densidad de los nodos de equipos móviles con transmisión de onda corta,

- Bettencourt et al $(2008,3)$ con el estudio de la difusión de los diagramas de Feynman en las comunidades de físicos teóricos de Estados Unidos, Japón y la URSS, en el período posterior a la Segunda Guerra Mundial, con el fin de determinar cómo los diagramas de Feynman pasan de ser una herramienta computacional de la física teórica, a un uso extensivo en otras áreas, como la física atómica y la teoría de la materia condensada,

- Urbizagástegui Alvarado $(2008,91)$ en el campo de la Ciencia de la Información aplica la teoría epidémica a la literatura sobre la Ley de Lotka, para verificar la existencia de una epidemia de los autores de artículos científicos sobre la productividad de los autores o ley de Lotka,

- Yan y Gerstein $(2011,1)$ en un estudio de Altmetría lo utilizan para determinar la difusión de la información científica, mediante el uso y el archivo de páginas web pertenecientes a PloS (Public Library of Science) y sus 6 revistas temáticas: PloSBiology, PloSComputationalbiology, PloSGenetics, PloS Medicine, PloSOne y Pathogens.

\section{- Ventajas y limitaciones de la teoría epidémica}

Una de las ventajas de la teoría epidémica la brinda el autor Worthen $(1973,227)$ quien afirma que la aplicación del modelo epidémico al estudio de la literatura en un campo específico permite descubrir si está en una fase de crecimiento, declive o estabilidad, y que se derivan otras observaciones como el cambio de énfasis en la investigación o la introducción de una idea nueva en el dicha área. Esto se ve confirmado por el análisis de Goffman $(1971,180)$ aplicado al estudio de la lógica simbólica al observar que, en la comparación de los autores infectados y 
removidos, se constata que ocurre una epidemia cada 25 años y se registra el descubrimiento de una idea cada 12,5 años.

Goffman y Newill (1964, 227-228) plantean que la aplicación de su método al estudio del crecimiento científico sirve para responder varias preguntas que son fundamentales para la implantación y manejo de un sistema de recuperación de información. Las preguntas que pueden ser respondidas son las siguientes:

1- ¿Cuál es el momento más oportuno para introducir un sistema de recuperación de información en una comunidad de científicos?

2- ¿Dónde y cuándo estará una disciplina científica desarrollándose en proporciones epidémicas?

3- ¿Cuál es la duración estimada de esta actividad epidémica?

4- ¿Cuál es la intensidad de esta epidemia?

5- ¿Cuáles son los trabajos principales de una disciplina que están diseminando una idea?

En un centro de Información o Biblioteca las respuestas a estas preguntas tienen que ver con la planificación de la colección al poder predecir la emergencia de una actividad en un área, su duración, su tamaño, la importancia relativa y la intensidad de ese asunto. Podrán indicar, además de bases cuantitativas para una política de adquisición, una guía para el establecimiento de prioridades de servicios y prever cambios en la producción de literatura, en su publicación y uso.

Hay algunas limitaciones de la teoría epidémica que deberían ser objeto de mayores estudios (Oliveira 1984, 289). Una se refiere al tipo de documentoincluido en los estudios epidémicos en el que no se considera la comunicación informal y se toma la lectura como agente transmisor de una infección o una idea. Si se toma en cuenta que hay múltiples formas de adquirir una idea, además de la lectura, la teoría epidémica no puede explicar por qué medios fue realmente infectado un individuo.

Otra debilidad de esta teoría es el período de tiempo a considerar en los estudios de las disciplinas. La falta de límites en el período, así como en el número de publicaciones o de autores son factores que pueden afectar los resultados finales. Uno de los aspectos que plantean un desafío tiene que ver, no solo con la teoría epidémica, sino en general con los estudios cuantitativos o bibliométricos. La utilización de los métodos cuantitativos para medir el volumen de la producción científica no permite evaluar directamente la calidad de esta producción nisus efectos en la sociedad.

- Concepto de epidemia y sus modelos matemáticos

Según la OPS $(2001,697)$ una epidemia se define como la "manifestación, en una comunidad o región, de casos de una enfermedad (o un brote) con una frecuencia que exceda netamente de la incidencia normal prevista". Esto significa que la epidemicidad no se define por un número absoluto de casos en cualquier lugar, momento o población. La aparición de un solo caso de una enfermedad transmisible que invade por primera vez una región o que no se constataba por un tiempo prolongado es suficiente para iniciar una investigación epidemiológica.

Una forma de entender la propagación de las infecciones es a través de la modelación matemática de las epidemias. En 1927 Kermack y McKendric 
publican el artículo titulado "A contribution to the mathematical theory of epidemics", en el que introducen un modelo matemático para las epidemias que tomaba en cuenta los aspectos de infección y remoción. En el caso del modelo de Kermack y McKendric los mecanismos involucrados que buscan representar se dividen en tres clases: el número de individuos susceptibles $\mathrm{S}(\mathrm{t})$, es decir, aquellos que pueden contagiarse; los infecciosos $\mathrm{I}(\mathrm{t})$, o los que al estar enfermos pueden contagiar la enfermedad; los removidos $\mathrm{R}(\mathrm{t})$, aquellos que después de enfermarse y aliviarse quedan en un estado de inmunidad, o bien están aislados en algún sitio (como en un hospital), o fallecieron. El escenario que se le presenta a un individuo puede ser descrito por el modelo SIR estándar: S-I-R.

\section{- Sustentabilidad: entre equívocos y ambigüedades}

El origen del concepto sustentabilidad tiene una larga historia que se remonta a un libro editado en 1713 llamado "Sylviculturao economica: instrucciones para el cultivo natural de árboles", del autor Hans Carl von Carlowitz (Schmithusen2013, 3) inspector jefe de minas de Sajonia. La relevancia histórica de este documento, es que Carlowitz fue el primero en asociar la palabra sustainability a la idea del cuidado y la previsión, y así hacer comprensible la idea de responsabilidad en el manejo presente para las futuras generaciones. El precursor del vocablo sostenible en el idioma alemán de la época es pfleglich (cuidadoso) y Carlowitz estableció la regla máxima: se debe administrar cuidadosamente la madera. El consumo de madera debe hacerse en el marco de lo que ese espacio físico es capaz de crear y soportar, de forma que el uso posterior pueda tener las siguientes características: permanente, continuado y perpetuo.

Brown et al $(1987,717)$ en su artículo "Global sustainability: towarddefinition”, presentan los elementos esenciales para definir la sustentabilidad:

- el apoyo continuado de la vida humana en la tierra,

- la conservación a largo plazo de los recursos biológicos y la productividad de los sistemas agrícolas,

- una población humana estable,

- economías con crecimiento limitado,

- calidad del ambiente y los ecosistemas.

El enfoque de los estudios sobre sustentabilidad se centra, mayoritariamente, en los problemas ambientales de forma global (Latchinian 2010, 112), y la discusión de la escala a ser aplicada (local o global) determina el tipo de problemas percibidos, así como sus soluciones.

La lista de problemas ambientales globales va variando de acuerdo a la agenda de los organismos internacionales, a datos científicos, y a la intensidad y urgencia de los mensajes de los medios de comunicación. En esta categoría o dominio se pueden considerar perturbaciones ambientales como la degradación de la capa de ozono, el cambio climático, la pérdida de biodiversidad, la lluvia ácida, la deforestación.

- La sustentabilidad y el desarrollo sustentable o sostenible.

Antes de plantear la relación entre los conceptos de sustentabilidad y desarrollo sostenible, es necesaria una explicación acerca de los adjetivos sostenible y sustentable. Cuando se propuso por primera vez el término desarrollo sustentable 
fue en el idioma original del Informe Brundtland (Naciones Unidas, 1987), en inglés. Al traducirlo al español surgieron discusiones acerca de cuál era el adjetivo más adecuado si sostenible o sustentable. El Diccionario de la Real Academia Española (2006) define sostenible como "el proceso que puede mantenerse por sí mismo", y sustentable, al derivarse de sustentar como "proveer a alguien del alimento necesario y conservar algo en su ser o estado". Si bien se pueden utilizar ambos términos como sinónimos, y de hecho se lo hace, el que mejor da cuenta del proceso de cuidado de los recursos presentes para su uso en el futuro es el adjetivo sostenible.

En cuanto a la diferenciación entre los términos sustentabilidad y desarrollo sostenible, el primero es un concepto más amplio. El primero refiere a "la condición o estado que permitiría la continuación indefinida de la existencia de la especie humana en la Tierra, mediante una vida sana, segura, productiva y en armonía con la naturaleza" (López, 2006). El segundo tiene que ver con el crecimiento que procura mantener un equilibrio dinámico entre la demanda de equidad, prosperidady una mejor calidad de vida de las individuos, así como lo que es ecológicamente posible en términos de recursos.

Las características enunciadas por Carlowitz para la administración de la madera (permanente, continuado y perpetuo) son las que toma y difunde el reporte "Nuestro futuro común" de las Naciones Unidas en 1987, también llamado reporte Brundtland (por la doctora noruega Gro Harlem Brundtland que presidía la Comisión Mundial sobre Medio Ambiente y Desarrollo) al enunciar: "Está en manos de la humanidad hacer que el desarrollo sea sostenible, es decir, asegurar que satisfaga las necesidades del presente sin comprometer la capacidad de las futuras generaciones para satisfacer las propias. El concepto de desarrollo sostenible implica límites, no límites absolutos, sino limitaciones que imponen a los recursos del medio ambiente el estado actual de la tecnología y de la organización social, y la capacidad de la biósfera de absorber los efectos de las actividades humanas, pero tanto la tecnología como la organización social pueden ser ordenadas y mejoradas de manera que abran el camino a una nueva era de crecimiento económico." (Naciones Unidas, 1987, 23).

La focalización de la atención en el Desarrollo Sostenible o Sustentable por parte de los organismos internacionales impulsó que se tomara en cuenta, por parte de los planificadores y ejecutores, de las políticas públicas, así como por la comunidad científica internacional (Goodland 1995, 20). También en las publicaciones hay un incremento del área sustentabilidad y se registra un número creciente de institutos que adoptaron el desarrollo sostenible como su foco de investigaciones e incluso se establecieron cursos de grado. Por ejemplo, la Universidad de Arizona fundó la Escuela de Sustentabilidad (2006), y la Universidad de Tokio tiene un programa en Ciencia de la Sustentabilidad (2007). Otras universidades han creado redes de investigación en el área: Universidad de Hokkaido, de Rikkyo y Osaka.

\section{METODOLOGÍA}

Para alcanzar los objetivos de la investigación se aborda el análisis de los autores que escriben sobre sustentabilidad desde la perspectiva de la bibliometría, utilizando sus técnicas y herramientas. 
Las características de este estudio son las de ser descriptivo y longitudinal. La característica de descriptivo implica que se busca describir situaciones, hechos o personas, midiéndolos y evaluándolos de acuerdo a las dimensiones o variables especificadas previamente.La longitudinalidad del estudio está dada porque los datos se recolectarán a través del tiempo, tomando en cuenta períodos de tiempo para inferir cambios que se hayan producido en la literatura estudiada.

\section{- Selección de la fuente de datos}

Se decidió trabajar con el método de cuenta completa, esto significa que al asignar el crédito de los documentos producidos por varios autores, se asigna el documento a cada uno de los autores. Si el documento tiene tres autores, cada uno recibe el crédito completo. El método de cuenta completa garantiza que todos los autores sean contabilizados, independientemente de su lugar en el grupo de autores.

La recolección de los datos se hizo tomando las referencias bibliográficas que incluyen el tema "sustentabilidad" en las áreas de estudio de la Ecología y el Ordenamiento territorial en la plataforma de información internacional Web of Science.

Las búsquedas se realizaron en la Web of Science en dos de sus bases de datos: Science Citation Index Expanded (SCI-EXPANDED) y Social Sciences Citation Index (SSCI) el 6 de febrero de 2017.

Las ventajas de realizar la investigación en la plataforma Web of Science son que tiene carácter internacional y multidisciplinar, tiene una amplia cobertura de las publicaciones más importantes en las diferentes áreas del conocimiento, es actualizada periódicamente, los registros son completos, y permite la exportación de los resultados de búsqueda a múltiples formatos que facilitan su análisis.

\section{- Delimitación del área y período de estudio}

El lapso de tiempo a estudiar va desde 1987 al 2015. Fue en el año 1987 cuando se hizo público el reporte Brundtland, provocando una diseminación mayor del término sustentabilidad. La cobertura geográfica es la determinada por la inclusión de las revistas a las bases de datos, siendo esperable un menor número de publicaciones científicas de los países subdesarrollados o con menor inversión en investigación científica y tecnológica.

La elección de las áreas de Ecología y Ordenamiento territorial fue realizada tomando en cuenta su confluencia teórica, concibiendo al territorio como recurso y factor de desarrollo y no sólo como soporte físico para las actividades y los procesos económicos (Troitiño 2006, 21).

- $\quad$ Tipos de documentos

Los documentos seleccionados en la búsqueda fueron los artículos y revisiones, excluyéndose las cartas de autores y resúmenes de libros.

- Tratamiento de los datos 
En la definición de la estrategia de búsqueda se determinó el término por el cual se realizaría, adoptando el término de "sustainability" que abarca los conceptos sustentabilidad y sostenibilidad que se utilizan en el idioma castellano. En el análisis de los datos, discusión y conclusiones se utilizó sustentabilidad y sostenibilidad como sinónimos. Se realizó el sondeo en la interfaz de búsqueda básica en las bases de datos de Web of Sciencede Science Citation Index Expanded (SCI-EXPANDED) y Social Sciences Citation Index (SSCI) por el término "sustainability" en el campo de tema. Se refinó la búsqueda por el período de tiempo desde el año 1987 al 2015 a nivel internacional.

Posteriormente, se realizó la refinación de resultados por las categorías de la Web of Science que dan cuenta de las áreas de Ecología y Ordenamiento territorial, ellas son: Environmental Science, Environmental Studies, Ecologyy Urban Studies.

Para calcular los autores infectados se eliminaron los autores a partir de su segundo trabajo y se contaron la cantidad de autores totales por año. La tasa de crecimiento anual de los infectados se calculó en base a la siguiente fórmula:

$$
\mathrm{f}-\mathrm{s} / \mathrm{s}
$$

donde $\mathrm{f}$ es el dato del último año y s es el dato del año anterior al considerado.

Para determinar los autores removidos se tomaron aquellos a partir de un año posterior a la última publicación. La tasa de crecimiento de removidos se calculó en base a la siguiente fórmula:

$$
\mathrm{f}-\mathrm{s} / \mathrm{s}
$$

donde $\mathrm{f}$ es el dato del último año y s es el dato del año anterior al considerado.

Los autores susceptibles se determinaron tomando dos fuentes de información: los autores que hicieron comentarios al Informe Brundtland (Sciandro 2010, 5), y la cantidad de estudiantes inscriptos en la Universidad de Wisconsin en 1987, donde eran docentes los cuatro autores del primer artículo en el período estudiado (US National Center for EducationStatistics1993, 52). El total de autores susceptibles quedó definido en 1000.

- Análisis estadístico de los datos

El sistema de ecuaciones diferenciales presentado por Goffman es el siguiente:

$\mathrm{dS} / \mathrm{dt}=-\beta \mathrm{SI}$

$\mathrm{dI} / \mathrm{dt}=\beta \mathrm{SI}-\gamma \mathrm{I}$

$\mathrm{dR} / \mathrm{dt}=\gamma \mathrm{I}$

donde $\mathrm{S}$, I y $\mathrm{R}$ son funciones continuas de la variable $\mathrm{t}$ (tiempo), $\beta$ es la tasa de infección de los susceptibles, $\gamma$ es la es la tasa a la cual los infectados son removidos.

El sistema de ecuaciones diferenciales no tiene una solución numérica, para resolver esto se utilizó la ecuación de Hurewicz $(1958,25)$ que en forma de vector propone la siguiente fórmula: $\mathrm{X}_{\mathrm{i}-1} 1+[\mathrm{t}-\mathrm{t} \mathrm{i}-1] \mathrm{Xi}-1$

Lo que significa la fórmula es que la tasa de cambio de infectados en un determinado tiempo será igual a la tasa de cambio de infectados del período 
anterior más la diferencia entre los dos períodos multiplicado por la tasa de cambio de infectados del período anterior.

\section{RESULTADOS Y DISCUSIÓN}

- Análisis sobre la producción de artículos de sustentabilidad

En los años estudiados se observa, mayoritariamente, un crecimiento en la cantidad de artículos publicados por año sobre sustentabilidad, completando un total de 16.563 artículos.

En términos porcentuales, los mayores incrementos se dan en los años 1990, 1988, 2013 y 2008 con 420\%, $200 \%, 101 \%$ y 95\% respectivamente.

La disminución en la publicación de artículos sobre sustentabilidad, con respecto al año anterior, se registra en los años 1999, 2004, 2007 y 2012, siendo el 2007 donde el porcentaje es menor $(-30 \%)$.

Gráfico 1. Cantidad de artículos por año

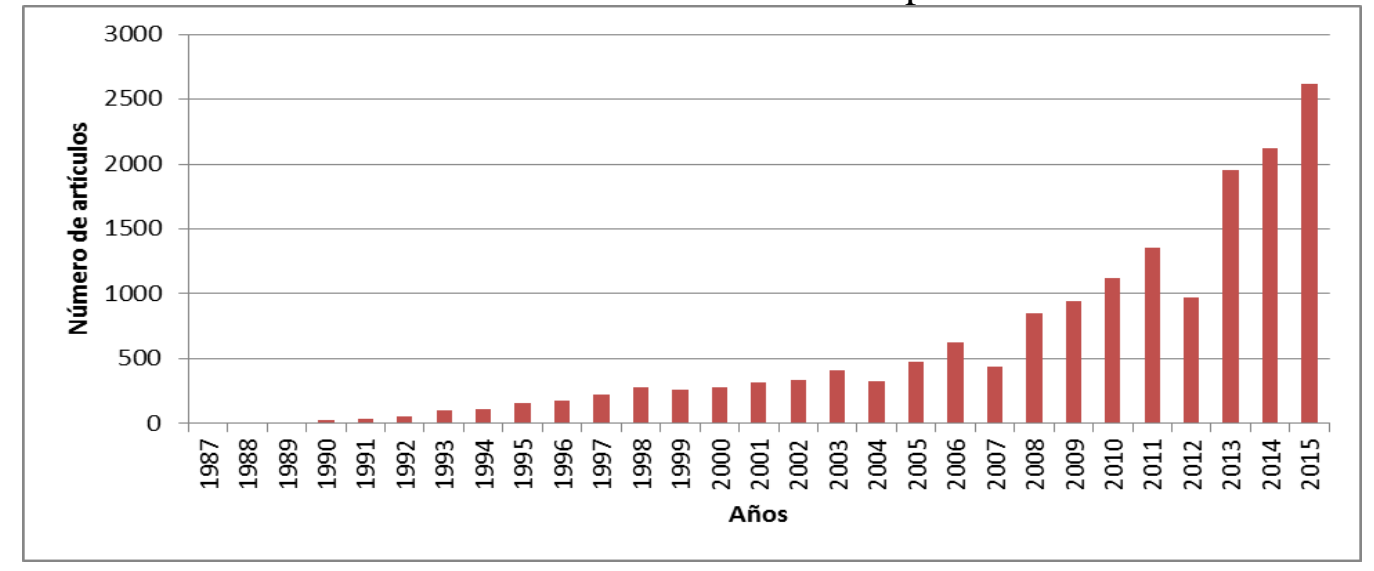

- Análisis de la producción de artículos de sustentabilidad por países

La cantidad de países a los que pertenecen los autores que escribieron sobre sustentabilidad es de 135 .

Estados Unidos se presenta como el país al que pertenecen la mayoría de los autores, con el $22 \%$. Los países que le siguen tienen un porcentaje mucho menor: por ejemplo, los que pertenecen al Reino Unido son un $11 \%$ del total, Australia un $7 \%$, y China un $6 \%$.

Del total de 135 países, 23 reúnen el 86,93 \% de los artículos, indicando una concentración elevada de la producción científica en pocos países.

En el cuadro siguiente se pueden apreciar los 23 países que reúnen la mayoría de artículos:

Cuadro 3. Cantidad de artículos por país

\begin{tabular}{|l|l|l|}
\hline País & Artículos & Porcentaje \\
\hline ESTADOS UNIDOS & 3629 & $21,91 \%$ \\
\hline REINO UNIDO & 1802 & $10,88 \%$ \\
\hline
\end{tabular}




\begin{tabular}{|l|l|l|}
\hline AUSTRALIA & 1114 & $6,73 \%$ \\
\hline CHINA & 968 & $5,84 \%$ \\
\hline CANADA & 852 & $5,14 \%$ \\
\hline ALEMANIA & 776 & $4,69 \%$ \\
\hline PAISES BAJOS & 670 & $4,05 \%$ \\
\hline ITALIA & 610 & $3,68 \%$ \\
\hline ESPAÑA & 573 & $3,46 \%$ \\
\hline SUECIA & 487 & $2,94 \%$ \\
\hline FRANCIA & 352 & $2,13 \%$ \\
\hline BRASIL & 312 & $1,88 \%$ \\
\hline SUIZA & 280 & $1,69 \%$ \\
\hline INDIA & 260 & $1,57 \%$ \\
\hline JAPON & 238 & $1,44 \%$ \\
\hline TURQUIA & 202 & $1,22 \%$ \\
\hline SUDAFRICA & 200 & $1,21 \%$ \\
\hline NUEVA ZELANDA & 190 & $1,15 \%$ \\
\hline AUSTRIA & 186 & $1,12 \%$ \\
\hline BELGICA & 183 & $1,10 \%$ \\
\hline PORTUGAL & 178 & $1,07 \%$ \\
\hline GRECIA & 170 & $1,03 \%$ \\
\hline DINAMARCA & 166 & $1,00 \%$ \\
\hline
\end{tabular}

En el mapa puede apreciarse más claramente la producción de los autores tomando como referencia a los países donde se desempeñan.

Gráfico 2. Representación geográfica de las publicaciones de Sustentabilidad en el período 1987-2015

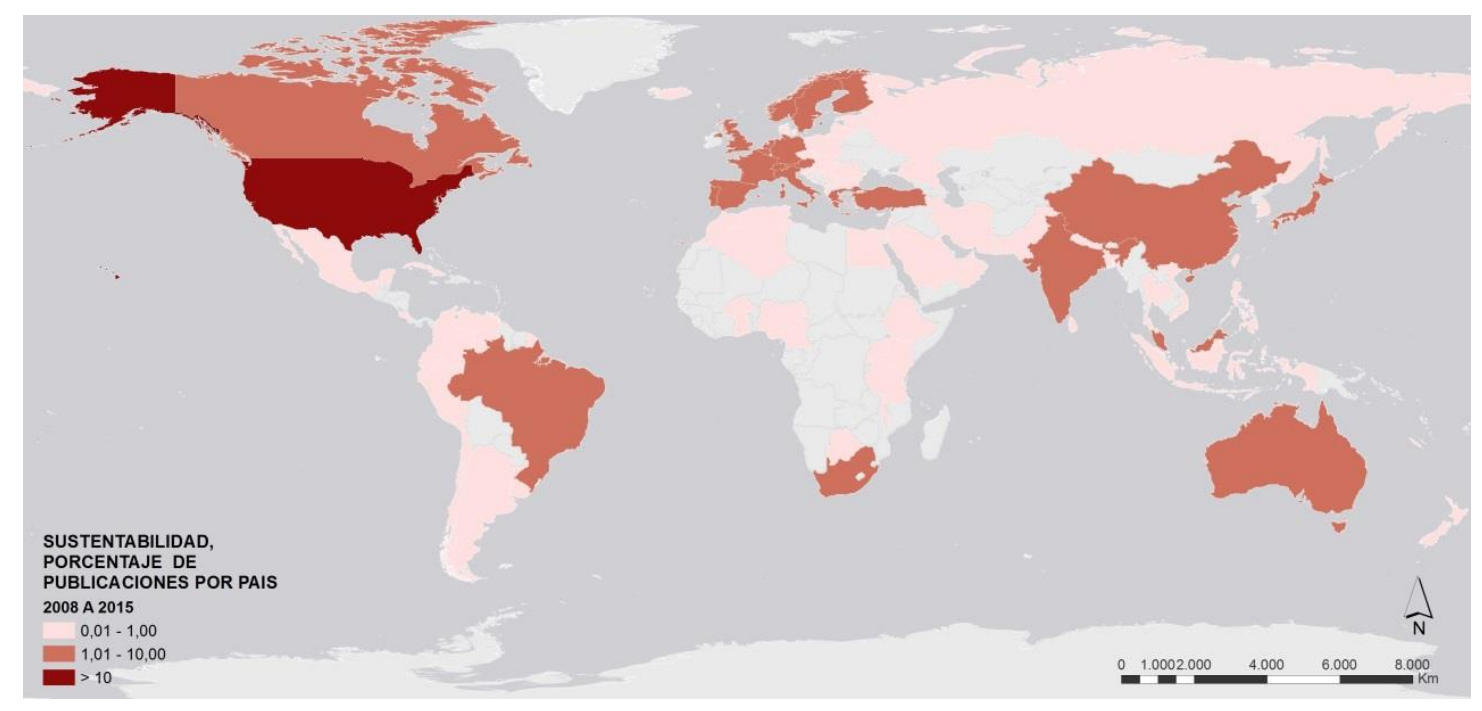

- Análisis de la producción sobre sustentabilidad por idioma

El idioma en que escriben los autores es casi en su totalidad el inglés, si bien en el último período (desde el 2008 al 2015) se incluyen artículos en otros idiomas: alemán, español, francés, polaco, portugués, turco (gráfico 3). 
Gráfico 3. Idiomas de los artículos sobre sustentabilidad

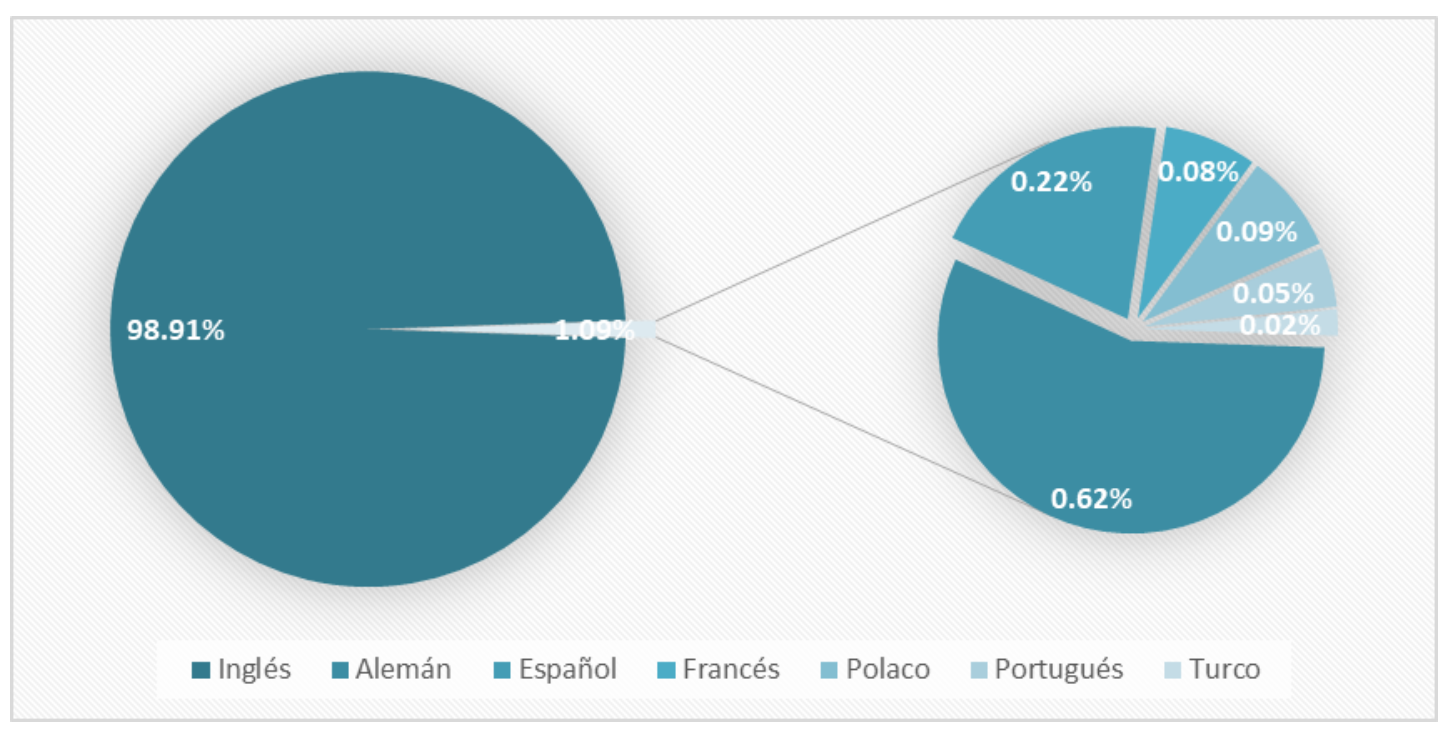

- Análisis epidémico de la producción sobre sustentabilidad

El total de artículos sobre sustentabilidad en el período 1987-2015 es de 16.563, donde la tasa de artículos por autor se centra en la mayoría de los años entre 0,40 y 0,70. Las excepciones se producen en el año 1987, con una tasa de 0,25 y en 1988 con 1,5 (Cuadro 4).

Cuadro 4. Artículos de sustentabilidad, autores y tasa de artículos/ autores

\begin{tabular}{|l|l|l|l|}
\hline Año & Artículos & Autores & Tasa de artículos/autores \\
\hline $\mathbf{1 9 8 7}$ & 1 & 4 & 0,25 \\
\hline $\mathbf{1 9 8 8}$ & 3 & 2 & 1,50 \\
\hline $\mathbf{1 9 8 9}$ & 5 & 7 & 0,71 \\
\hline $\mathbf{1 9 9 0}$ & 26 & 43 & 0,60 \\
\hline $\mathbf{1 9 9 1}$ & 32 & 61 & 0,52 \\
\hline $\mathbf{1 9 9 2}$ & 53 & 78 & 0,68 \\
\hline $\mathbf{1 9 9 3}$ & 97 & 133 & 0,73 \\
\hline $\mathbf{1 9 9 4}$ & 112 & 161 & 0,70 \\
\hline $\mathbf{1 9 9 5}$ & 156 & 267 & 0,58 \\
\hline $\mathbf{1 9 9 6}$ & 173 & 333 & 0,52 \\
\hline $\mathbf{1 9 9 7}$ & 221 & 377 & 0,59 \\
\hline $\mathbf{1 9 9 8}$ & 281 & 485 & 0,58 \\
\hline $\mathbf{1 9 9 9}$ & 257 & 462 & 0,56 \\
\hline $\mathbf{2 0 0 0}$ & 279 & 532 & 0,52 \\
\hline $\mathbf{2 0 0 1}$ & 315 & 591 & 0,53 \\
\hline $\mathbf{2 0 0 2}$ & 338 & 625 & 0,54 \\
\hline $\mathbf{2 0 0 3}$ & 409 & 868 & 0,47 \\
\hline $\mathbf{2 0 0 4}$ & 329 & 705 & 0,47 \\
\hline $\mathbf{2 0 0 5}$ & 474 & 996 & 0,48 \\
\hline $\mathbf{2 0 0 6}$ & 622 & 1307 & 0,48 \\
\hline & & & \\
\hline & 32 & 43 & 4 \\
\hline
\end{tabular}


Cont. Cuadro 4. Artículos de sustentabilidad, autores y tasa de artículos/ autores

\begin{tabular}{|l|l|l|l|}
\hline Año & Artículos & Autores & Tasa de artículos/autores \\
\hline $\mathbf{2 0 0 7}$ & 438 & 978 & 0,45 \\
\hline $\mathbf{2 0 0 8}$ & 854 & 1834 & 0,47 \\
\hline $\mathbf{2 0 0 9}$ & 939 & 2122 & 0,44 \\
\hline $\mathbf{2 0 1 0}$ & 1117 & 2472 & 0,45 \\
\hline $\mathbf{2 0 1 1}$ & 1356 & 3190 & 0,43 \\
\hline $\mathbf{2 0 1 2}$ & 972 & 2309 & 0,42 \\
\hline $\mathbf{2 0 1 3}$ & 1956 & 4446 & 0,44 \\
\hline $\mathbf{2 0 1 4}$ & 2126 & 5026 & 0,42 \\
\hline $\mathbf{2 0 1 5}$ & 2622 & 6150 & 0,43 \\
\hline
\end{tabular}

El gráfico 4 muestra el promedio de publicaciones por autor a través del tiempo. Se puede apreciar que las cifras después de cierta inestabilidad en los primeros años tienden a estabilizarse en el entorno de dos autores por cada artículo, siendo este promedio inferior que el nivel internacional en el mismo campo (Universidad de Granada, 2009). El crecimiento de la literatura en esta temática está determinado por el crecimiento del número de autores que actúan en el campo, y a la vez por el aumento de su producción.

Gráfico 4. Promedio de publicaciones por autor

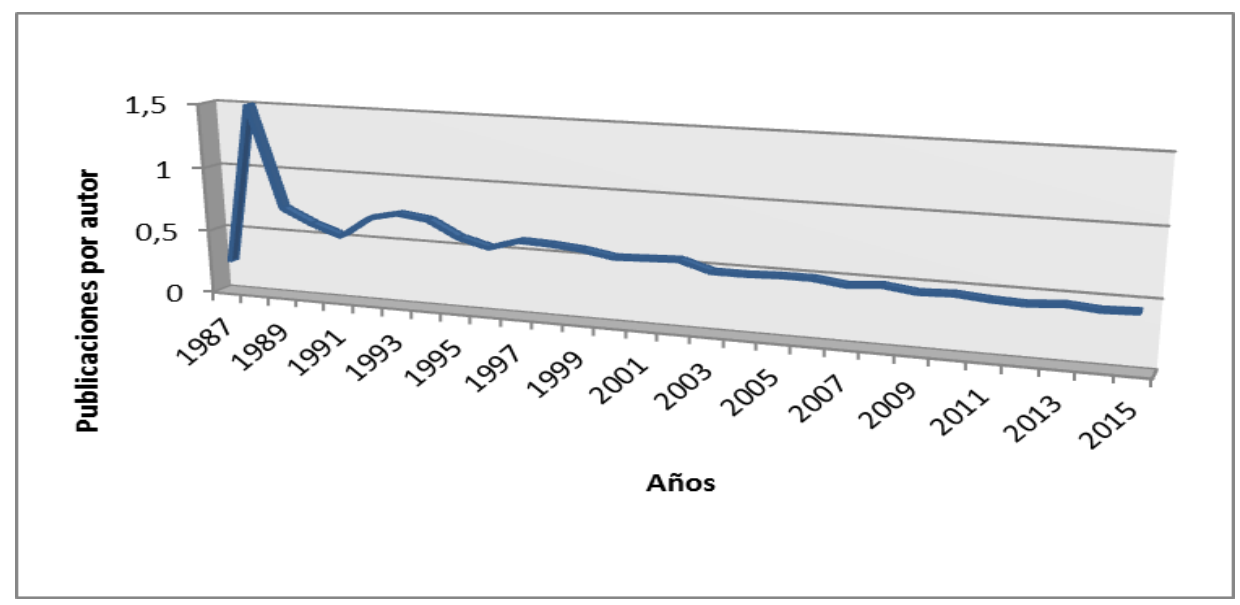

De acuerdo a la ecuación de Goffman hay una ocurrencia de epidemia si el resultado de $\beta-\gamma / \mathrm{S}=>0$, o sea si el índice de infección menos el índice de remoción sobre los susceptibles es mayor a 0 .

El proceso epidemiológico puede ser representado por el sistema de ecuaciones diferenciales utilizado por Goffman, que es el siguiente:

$\mathrm{dS} / \mathrm{dt}=-\beta \mathrm{SI}$

$\mathrm{dI} / \mathrm{dt}=\beta \mathrm{SI}-\gamma \mathrm{I}$

$\mathrm{dR} / \mathrm{dt}=\gamma \mathrm{I}$

donde $\mathrm{S}$, I y R son funciones continuas de la variable $\mathrm{t}$ (tiempo), $\beta$ es la tasa de infección de los autores considerados susceptibles, $\gamma$ es la es la tasa a la cual los 
autores infectados son removidos. Para que el proceso sea considerado epidémico el resultado de $\beta-\gamma / \mathrm{S}$ debe ser mayor que 0 .

Como se observa en el cuadro 5, el proceso es epidémico, o sea el resultado es mayor a 0 en los años 1993, 1995, 1998, 2003, 2005, 2008, 2011 y 2013.

El resultado es igual a 0 en los años 1987 al 1992, 2000 y 2002.

El resultado es negativo en los años 1994, 1996, 1997, 1999, 2001, 2004, 2006, 2007, 2009, 2010, 2012, 2014 у 2015.

Cuadro 5. Ocurrencia de epidemia del término sustentabilidad

\begin{tabular}{|l|l|l|l|l|l|l|l|l|l|}
\hline Año & Inf. & DI & Rem. & DH & $\beta S I$ & Susc. & $\beta$ & $\gamma$ & $\begin{array}{l}\beta- \\
\gamma / \mathrm{S}\end{array}$ \\
\hline 1987 & 4 & 4 & 0 & 0 & 4 & 996 & 0,02 & 0 & 0 \\
\hline 1988 & 2 & -1 & 0 & 0 & -1 & 1001 & 0 & 0 & 0 \\
\hline 1989 & 7 & 0,7 & 5 & 1 & 0 & 1000 & 0 & 0,71 & 0 \\
\hline 1990 & 43 & 0,8 & 6 & 0,2 & 1 & 999 & 0,04 & 0,14 & 0 \\
\hline 1991 & 61 & 0,3 & 33 & 0,8 & $-0,5$ & 1001 & $-0,03$ & 0,54 & 0 \\
\hline 1992 & 78 & 0,2 & 47 & 0,3 & $-0,1$ & 1000 & $-0,01$ & 0,6 & 0 \\
\hline 1993 & 133 & 0,4 & 47 & 0 & 0,4 & 1000 & 0,06 & 0,35 & 0,1 \\
\hline 1994 & 161 & 0,2 & 100 & 0,5 & $-0,4$ & 1000 & $-0,06$ & 0,62 & $-0,1$ \\
\hline 1995 & 267 & 0,4 & 110 & 0,1 & 0,3 & 1000 & 0,08 & 0,41 & 0,1 \\
\hline 1996 & 333 & 0,2 & 176 & 0,4 & $-0,2$ & 1000 & $-0,06$ & 0,53 & $-0,1$ \\
\hline 1997 & 377 & 0,1 & 254 & 0,3 & $-0,2$ & 1000 & $-0,07$ & 0,67 & $-0,1$ \\
\hline 1998 & 485 & 0,2 & 279 & 0,1 & 0,1 & 1000 & 0,06 & 0,58 & 0,1 \\
\hline 1999 & 462 & 0 & 336 & 0,2 & $-0,2$ & 1000 & $-0,1$ & 0,73 & $-0,1$ \\
\hline 2000 & 532 & 0,1 & 368 & 0,1 & 0 & 1000 & 0,02 & 0,69 & 0 \\
\hline 2001 & 591 & 0,1 & 452 & 0,2 & $-0,1$ & 1000 & $-0,05$ & 0,76 & $-0,1$ \\
\hline 2002 & 625 & 0,1 & 451 & 0 & 0,1 & 1000 & 0,04 & 0,72 & 0 \\
\hline 2003 & 868 & 0,3 & 484 & 0,1 & 0,2 & 1000 & 0,18 & 0,56 & 0,2 \\
\hline 2004 & 705 & $-0,2$ & 686 & 0,3 & $-0,5$ & 1001 & $-0,37$ & 0,97 & $-0,4$ \\
\hline 2005 & 996 & 0,3 & 572 & $-0,2$ & 0,5 & 1000 & 0,49 & 0,57 & 0,5 \\
\hline 2006 & 1307 & 0,2 & 810 & 0,3 & $-0,1$ & 1000 & $-0,07$ & 0,62 & $-0,1$ \\
\hline 2007 & 978 & $-0,3$ & 1034 & 0,2 & $-0,6$ & 1001 & $-0,54$ & 1,06 & $-0,5$ \\
\hline 2008 & 1834 & 0,5 & 782 & $-0,3$ & 0,8 & 999 & 1,45 & 0,43 & 1,4 \\
\hline 2009 & 2122 & 0,1 & 1575 & 0,5 & $-0,4$ & 1000 & $-0,78$ & 0,74 & $-0,8$ \\
\hline 2010 & 2472 & 0,1 & 1910 & 0,2 & 0 & 1000 & $-0,08$ & 0,77 & $-0,1$ \\
\hline 2011 & 3190 & 0,2 & 2204 & 0,1 & 0,1 & 1000 & 0,29 & 0,69 & 0,3 \\
\hline 2012 & 2309 & $-0,4$ & 3149 & 0,3 & $-0,7$ & 1001 & $-1,57$ & 1,36 & $-1,6$ \\
\hline 2013 & 4446 & 0,5 & 2692 & $-0,2$ & 0,7 & 999 & 2,89 & 0,61 & 2,9 \\
\hline 2014 & 5026 & 0,1 & 4971 & 0,5 & $-0,3$ & 1000 & $-1,72$ & 0,99 & $-1,7$ \\
\hline 2015 & 6150 & 0,2 & 6650 & 0,3 & $-0,1$ & 1000 & $-0,43$ & 1,08 & $-0,4$ \\
\hline
\end{tabular}

Como se detalló en la descripción del proceso epidémico, el mismo puede presentar dos estados:

a) estable, cuando la tasa de variación del número de infectados con relación al tiempo es igual a la tasa de variación del número de removidos con relación al mismo tiempo, 
b) inestable, cuando la tasa de variación de número de infectados con relación al tiempo es diferente de la tasa de variación del número de removidos con relación al mismo tiempo.

En el caso de la epidemia sobre el concepto de sustentabilidad en la literatura científica el proceso se comportó de forma estable solo en el año 2000.

La inestabilidad del proceso se puede apreciar claramente en el gráfico 5.

Gráfico 5. Proceso epidémico del tema sustentabilidad

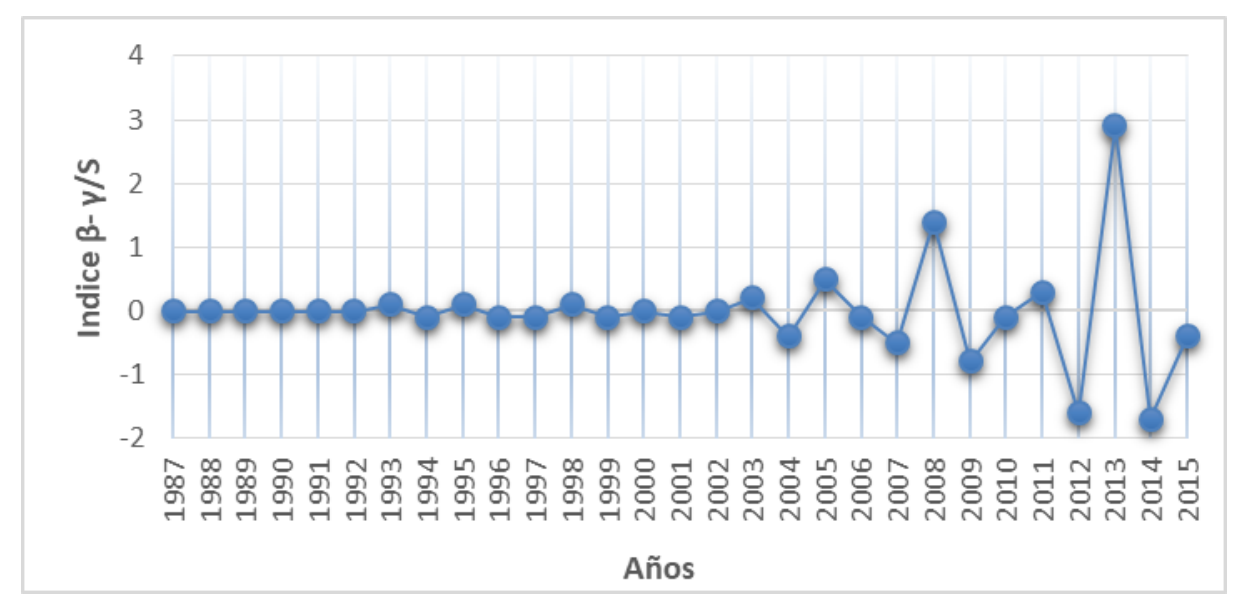

La inestabilidad del proceso epidémico demuestra el alto nivel de movilidad de los autores que pasan a publicar sobre el tema de sustentabilidad (y por lo tanto están infectados), así como los que dejan de publicar en el mismo período (los que se denominan autores removidos).

De acuerdo al modelo epidémico, los tres estados en los que pueden incluirse los autores son: infectados, removidos y susceptibles.

Para determinar los autores infectados se eliminaron los autores a partir de su segundo trabajo y se contaron la cantidad de autores totales por año. Para detectar los autores removidos se tomaron aquellos a partir de un año posterior a la última publicación, mientras que para determinar los autores susceptibles se tomaron dos fuentes de información: los autores que hicieron comentarios al Informe Brundtland (Sciandro 2010, 5), y la cantidad de estudiantes inscriptos en la Universidad de Wisconsin en 1987, donde eran docentes los cuatro autores del primer artículo en el período estudiado (US National Center for Education Statistics 1993, 52), quedando definido en 1000.

Cuadro 6. Autores susceptibles, infectados y removidos.

\begin{tabular}{|l|l|l|l|}
\hline Año & Autores infectados & Autores removidos & $\begin{array}{l}\text { Autores } \\
\text { susceptibles }\end{array}$ \\
\hline $\mathbf{1 9 8 7}$ & 4 & 0 & 996 \\
\hline $\mathbf{1 9 8 8}$ & 2 & 0 & 1000 \\
\hline $\mathbf{1 9 8 9}$ & 7 & 5 & 995 \\
\hline $\mathbf{1 9 9 0}$ & 43 & 6 & 994 \\
\hline $\mathbf{1 9 9 1}$ & 61 & 33 & 967 \\
\hline
\end{tabular}




\begin{tabular}{|l|l|l|l|}
\hline $\mathbf{1 9 9 2}$ & 78 & 47 & 953 \\
\hline $\mathbf{1 9 9 3}$ & 133 & 47 & 953 \\
\hline $\mathbf{1 9 9 4}$ & 161 & 100 & 900 \\
\hline $\mathbf{1 9 9 5}$ & 267 & 110 & 890 \\
\hline $\mathbf{1 9 9 6}$ & 333 & 176 & 824 \\
\hline $\mathbf{1 9 9 7}$ & 377 & 254 & 746 \\
\hline $\mathbf{1 9 9 8}$ & 485 & 279 & 721 \\
\hline $\mathbf{1 9 9 9}$ & 462 & 336 & 664 \\
\hline $\mathbf{2 0 0 0}$ & 532 & 368 & 632 \\
\hline $\mathbf{2 0 0 1}$ & 591 & 452 & 548 \\
\hline $\mathbf{2 0 0 2}$ & 625 & 451 & 549 \\
\hline $\mathbf{2 0 0 3}$ & 868 & 484 & 516 \\
\hline $\mathbf{2 0 0 4}$ & 705 & 686 & 314 \\
\hline $\mathbf{2 0 0 5}$ & 996 & 572 & 428 \\
\hline $\mathbf{2 0 0 6}$ & 1307 & 810 & 190 \\
\hline $\mathbf{2 0 0 7}$ & 978 & 1034 & -34 \\
\hline $\mathbf{2 0 0 8}$ & 1834 & 782 & 218 \\
\hline $\mathbf{2 0 0 9}$ & 2122 & 1575 & -575 \\
\hline $\mathbf{2 0 1 0}$ & 2472 & 1910 & -910 \\
\hline $\mathbf{2 0 1 1}$ & 3190 & 2204 & -1204 \\
\hline $\mathbf{2 0 1 2}$ & 2309 & 3149 & -2149 \\
\hline $\mathbf{2 0 1 3}$ & 4446 & 2692 & -1692 \\
\hline $\mathbf{2 0 1 4}$ & 5026 & 4971 & -3971 \\
\hline $\mathbf{2 0 1 5}$ & 6150 & 6650 & -5650 \\
\hline & 0 & & 1 \\
\hline
\end{tabular}

De acuerdo a Uribarri $(2009,240)$, la evolución típica de las variables SIR implica un aumento en el tiempo de la cantidad de infectados, una disminución del número de susceptibles, y en el caso de que el número de infectados descienda a 0 es que eventualmente se acaba la epidemia.

En el caso de los autores que escriben sobre sustentabilidad, en el gráfico 6 se puede observar que el proceso está en una curva ascendente. También se observa la inestabilidad del proceso epidémico, porque se presenta un número similar de autores que publican artículos sobre sustentabilidad (autores infectados) al de aquellos que dejan de publicar sobre el tema en el año de su último artículo (autores removidos). Esto indica que los autores que inician su producción científica con publicaciones sobre el tema de sustentabilidad representan una proporción tan alta como aquellos que dejan de hacerlo al año de su último artículo. 
Gráfico 6. Número de autores susceptibles, infectados y removidos

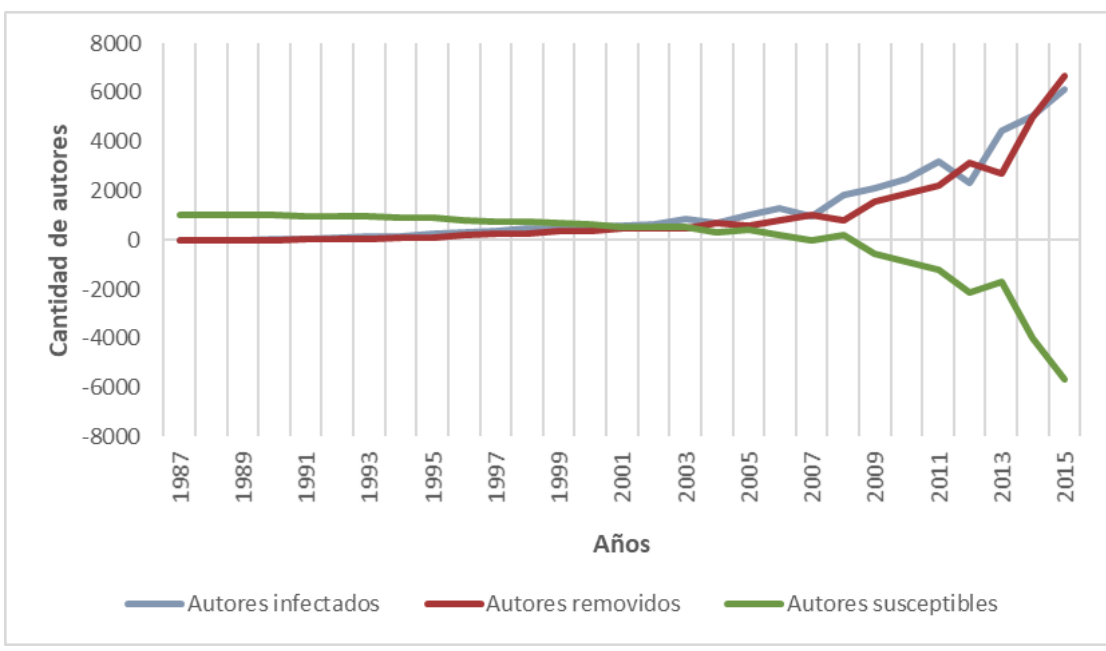

Tomando la expresión recursiva establecida por Hurewicz $(1958,25)$ y establecida en forma de vector, se puede calcular la estimación de autores infectados en los diferentes años.

El enunciado es el siguiente: $\mathrm{X}_{\mathrm{i}-1}+$ [t-t i-1] Xi-1.

Esto significa que la tasa de cambio de infectados en un tiempo dado será igual a la tasa de cambio de infectados en el período anterior más la diferencia entre los dos períodos, y multiplicado todo ello por la tasa de cambio de infectados del período anterior.

Los resultados de la aplicación de la ecuación presentada se aprecian en el siguiente cuadro.

Cuadro 7. Estimación de autores infectados

\begin{tabular}{|l|l|l|}
\hline Año & Datos estimados & Datos presentados \\
\hline $\mathbf{1 9 8 7}$ & & 4 \\
\hline $\mathbf{1 9 8 8}$ & 8 & 2 \\
\hline $\mathbf{1 9 8 9}$ & 4 & 7 \\
\hline $\mathbf{1 9 9 0}$ & 14 & 43 \\
\hline $\mathbf{1 9 9 1}$ & 86 & 61 \\
\hline $\mathbf{1 9 9 2}$ & 122 & 78 \\
\hline $\mathbf{1 9 9 3}$ & 156 & 133 \\
\hline $\mathbf{1 9 9 4}$ & 266 & 161 \\
\hline $\mathbf{1 9 9 5}$ & 322 & 267 \\
\hline $\mathbf{1 9 9 6}$ & 534 & 333 \\
\hline $\mathbf{1 9 9 7}$ & 666 & 377 \\
\hline $\mathbf{1 9 9 8}$ & 754 & 485 \\
\hline $\mathbf{1 9 9 9}$ & 970 & 462 \\
\hline $\mathbf{2 0 0 0}$ & 924 & 532 \\
\hline $\mathbf{2 0 0 1}$ & 1064 & 591 \\
\hline $\mathbf{2 0 0 2}$ & 1182 & 625 \\
\hline $\mathbf{2 0 0 9}$ & 3668 & 2122 \\
\hline
\end{tabular}


Cont. Cuadro 7. Estimación de autores infectados

\begin{tabular}{|l|l|l|}
\hline Año & Datos estimados & Datos presentados \\
\hline 2010 & $\mathbf{4 2 4 4}$ & 2472 \\
\hline 2011 & 4944 & 3190 \\
\hline 2012 & 6380 & 2309 \\
\hline 2013 & 4618 & 4446 \\
\hline 2014 & 8892 & 5026 \\
\hline 2015 & 10052 & 6150 \\
\hline
\end{tabular}

Como se aprecia claramente en el gráfico 7 , los datos estimados de autores siguen una curva similar durante todo el período de estudio, si bien en los 2 últimos años los autores estimados no se ajustan a los presentados.

Gráfico 7. Estimación del número de autores infectados

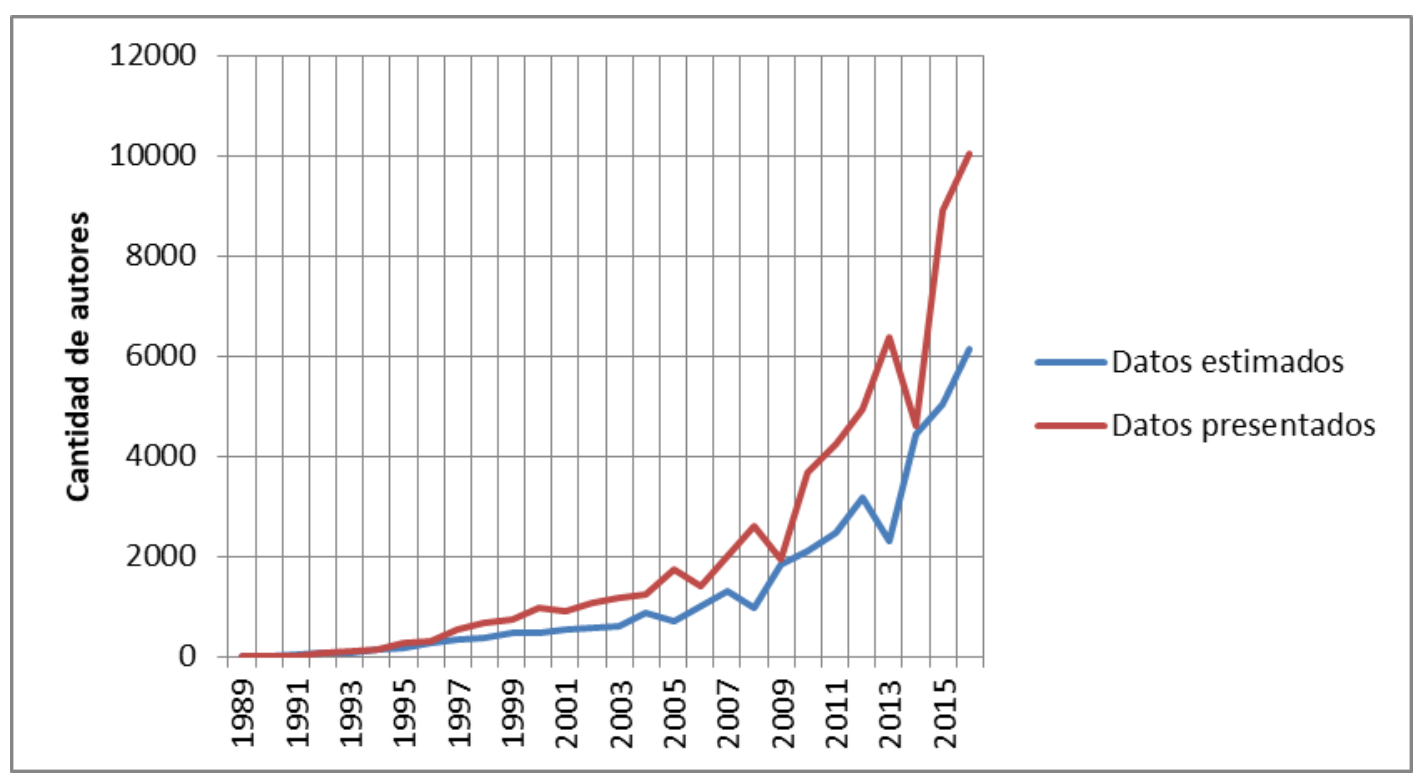

Los datos concuerdan con los presentados en otros trabajos, donde el patrón de crecimiento estimado de los artículos coincide con los datos presentados en las publicaciones, no siendo así la cantidad exacta de los mismos (Caldeira1975, 16; Urbizagástegui Alvarado2008, 105). Los hallazgos no son comparables con las investigaciones de Goffman, ya que sus investigaciones refieren al estudio del crecimiento de las subdisciplinas que integran un campo de estudio mayor (Goffman, 1966, 1971).

Para determinar el error de aproximación de la ecuación se calculó entonces el promedio de los errores absolutos y los errores relativos, medición de utilidad para determinar cuánto puede alejarse el resultado estimado al presentado. El error absoluto refiere a la diferencia entre el valor estimado y el valor medido, y el error relativo es el cociente entre el error absoluto y el valor exacto. El promedio de error absoluto calculado es de 866 y el de error relativo es de 1,7. 


\section{CONCLUSIONES}

Se exponen aquí las conclusiones obtenidas, tomando en cuenta los objetivos planteados, los resultados de la investigación y la discusión presentada a partir de ellos.

- El crecimiento sostenido de la producción científica sobre el tema sustentabilidad, durante el período 1987-2015, en las áreas de Ecología y Ordenamiento territorial en la base de datos Web of Science es constatable en cuanto a la cantidad de artículos por año, con una tasa promedio de 0,46. Tal incremento se mantiene excepto en los años 1999, 2004, 2007 y 2012.

- El crecimiento de la producción científica sobre el tema sustentabilidad se registra, además de la cantidad de artículos, de forma epidémica en la cantidad de autores. En tanto, en el número de países a los que pertenecen los autores, el promedio de crecimiento es de 59\% por cada periodo de 6 años. Referente a la cantidad de artículos de los diferentes temas tratados el crecimiento promedio es de $130 \%$.

- Al interpretar el conjunto de datos con el modelo epidémico y utilizando la ecuación de Goffman (con la resolución numérica que plantea Hurewicz) se advierte que el crecimiento de autores que escriben sobre sustentabilidad toma características epidémicas en los años 1993, 1995, 1998, 2003, 2005, 2008, 2011 y 2013. La ocurrencia de epidemia de acuerdo a Goffman se constata cuando el índice de infección menos el índice de remoción sobre los susceptibles es mayor a $0(\beta-\gamma / S=>0)$.

- El proceso epidémico de los autores que publican sobre el concepto de sustentabilidad se encuentra en una curva ascendente exponencial, por lo que cabe esperar que se produzca más literatura en esta área.

- Al comparar los datos obtenidos por la búsqueda y los estimados por el cálculo propuesto por la teoría epidémica, hay una alta similitud durante todo el período estudiado. El patrón de crecimiento de los autores que escriben artículos científicos sobre sustentabilidad pudo ser predicho por el modelo, si bien en los 2 últimos años la cantidad de autores estimados es superior a los presentados. Por lo tanto, se considera que la teoría epidémica es un marco conceptual adecuado para la interpretación del conjunto de datos sobre publicaciones de sustentabilidad. También es pertinente para la previsión de la curva o patrón de crecimiento de un campo científico y sus consecuencias en la documentación de Centros o Unidades de Información..

- Esta investigación ha constatado un patrón de crecimiento epidémico sobre el tema de sustentabilidad en la literatura científica. Este insumo podría tomarse para estudiar cualitativamente los motivos de ese crecimiento, así como los actores y factores implicados en él: desde el rol de los organismos internacionales, la realidad de una crisis ambiental con impactos económicos y sociales, hasta la ambigüedad y polisemia del término sustentabilidad. 


\section{Referencias Bibliográficas}

Banco Mundial (2017). Gasto en investigación y desarrollo (\% del PIB).

Recuperado de: https://datos.bancomundial.org/indicador/GB.XPD.RSDV.GD.ZS

Fecha de consulta: diciembre de 2017

Bennion, B, Neuton, L. (1976). The epidemiology of research on Anomalous water. En: Journal of the American Society for Information Science. 2(1):53-56

Bettencourt, L.M.A, Cintrón-Arias, A., Kaiser, D.I., Castillo-Chávez, C. (2008). The power of a good idea: quantitative modeling of the spread of ideas from epidemiological models. Recuperado de:

http://www.arxiv.org/abs/physics/0502067v3

Boff, L. (2014). Sustentabilidade. O que é, o que no é. Petrópolis: Vozes

Brown, B; Hanson, M; Liverman, D; Merideth, R. (1987). Global sustainability: toward definition. En: Environmental Management. 11(6):713-719

Caldeira, P (1975). Processo de crecimento epidemiológico aplicado á literatura brasileira de doenca de Chagas. En: Ci. Inf., Río de Janeiro. 4(1): 5-16

Comisión mundial del medio ambiente y del desarrollo. (1987). Nuestro futuro común. Madrid: Alianza.

Diamod, A.M. (1988). Thepolywater episode and the appraisal of theories. En: Donovan, A. et al (eds). Scrutinizing Science. Massachusetts: Kluwer. pp. 181198

Estados Unidos. Universidad de Arizona (2017). School of Sustainability.

Recuperado de: www.schoolofsustainability.asu.edu Fecha de consulta: $01 / 11 / 2017$

Goffman, W. (1966). Mathematical approach to the spread of scientific ideas The history of mast cell research. En: Nature. (5061): 449-452

Goffman, W. (1971). A mathematical method for analyzing the growth of a scientific discipline. En: Journal of the Association for Computing Machinery. 18(3): 173-185

Goffman, W, Newill, V. (1964), Generalization of epidemic theory: an application to the transmission of ideas En: Nature (4953): 225-228

Goodland, R. (1995). The concept of environmental sustainability. En: Annual Review of Ecology and Systematics. (26): 1-24

Hurewicz, W. (1958). Lectures on ordinary differential equations. Cambridge: MIT

Japón. Universidad de Tokio. (2017). Graduate program in Sustainability Science. Recuperado de: www.k.u-tokyo.ac.jp Fecha de consulta: 01/11/2017

Kermack, W.O., Mckendrick, A.G (1927). A contribution to the mathematical theory of epidemics. En: Proc. R. Soc. Lond. A 115, 700-721

Khelil, A, Becker, C, Tian, J, Rotherme, K. (2002) An epidemic model for information diffusion in MANETs. En: MSWiM: 1-7

Latchinian, A. (2010). Globotomía. Del ambientalismo mediático a la burocracia ambiental. Montevideo: Puntocero. 254 p. 
López, V.M. (2006). Sustentabilidad y Desarrollo Sustentable. México D.F.: Instituto Politécnico Nacional.

Oliveira, S. Marques de (1984). Estudo do comportamento da literatura brasileira de teologia adventista: análise de crescimento epidêmico. En: Ci. Inf., 13(1): 2552

Oliveira, S. Marques de (1984). Processo epidémico e processo de comunicacao. En: Rev. Bibliotecon. Brasilia, 12(2): 281-290

Organización Panamericana de la Salud. (2001) El control de las enfermedades transmisibles. Washington, OPS.

Real Academia Española. (2006). Diccionario de la Real Academia Española. Madrid: Espasa Calpe.

Sschmithusen, F. (2013). Three hundred years of applied sustainability in forestry. En: Unasylva 64: 3-11

Sciandro, J. (2010). Algunos apuntes sobre el nuevo marco legal del ordenamiento territorial vigente em Uruguay. Fórum de direito urbano e ambiental.

Troitiño, M (2006) Ordenación del territorio y desarrollo territorial: la construcción de las geografías del futuro. En: Cuadernos de Geografía 7(14): 1768

Universidad de Granada. (2019). Coauthor Index. Recuperado de: http://www.coauthorindex.info/layout.php?id=inicio

Urbizagástegui Alvarado, R y Suárez, J. (2008). La teoría epidémica en la literatura sobre la Ley de Lotka. En: Investigación Bibliotecológica. 22(46): 91111

Uribarri, S, Rodríguez Meza, M, Cervantes Cota, J. (2014) Las matemáticas de las epidemias: caso México 2009 y otros. En: Ciencia ergo-sum. 20(3): 238-246

Us National Center for Education Statistics. (1993). 120 years of american education: a statistical portrait. Washington: U.S. Department of Education Worthen, D. B. (1973) The epidemic process and the contagion model. En: Journal of the American Society for Information Science. 343-346

Yan, K.K, Gerstein, M. (2011) The spread of scientific information: insights from the web usage statistics in PloS article-level metrics. En: PloS ONE. 6(5): 1-7 